\title{
Trasladar las medidas de salud autopercibida a la consulta: un reto para el clínico
}

Rey E. Trasladar las medidas de salud autopercibida a la consulta: un reto para el clínico. An Med Interna (Madrid) 2007; $24: 413-414$.

La medición de los resultados en salud percibidos por el paciente (los "patient reported outcomes", los PRO, de los anglosajones) están adquiriendo una creciente importancia en todos los ámbitos de la medicina (1) por razones sociales, políticas y médicas (2) y han comenzado a ser valoradas por las agencias reguladoras para fundamentar determinados beneficios clínicos de fármacos (3) y son la razón de ser de ambiciosos proyectos como el PROMIS de los NIH (4). Pero, ¿para que queremos estos PRO si la medicina ha cumplido con los pacientes sin ellos durante siglos?

La medicina nació sobre la base de objetivos binarios (curación/no curación) pero, sobre todo a lo largo del siglo $\mathrm{XX}$, dio un salto importante con la incorporación de objetivos cuantitativos (medición y control de parámetros biológicos de la enfermedad), cuya importancia en la clínica es innegable e insustituible. Sin embargo, existen muchos problemas, enfermedades y situaciones cuyos efectos sobre la salud no son cuantificables mediante parámetros biológicos "objetivos" y que son relevantes, sobre todo si vemos la salud de persona desde un punto de vista global. La clásica pregunta ¿ha mejorado usted? sigue siendo útil en la clínica pero es difícil de trasladar a la evaluación de la eficacia de tratamientos. Por ejemplo, es relativamente fácil saber hasta que punto ha mejorado la capacidad para caminar de un paciente pero es más difícil estimar si ha disminuido el impacto de la enfermedad sobre su capacidad para trabajar o hasta que punto ha mejorado su sensación de bienestar. Estos elementos subjetivos de la salud se engloban en su mayoría dentro de lo que conocemos como calidad de vida relacionada con la salud, concepto cada vez más integrado en la investigación y la clínica y que muchos pacientes, especialmente los más jóvenes, ya empieza a solicitar en nuestra consulta como objetivos del tratamiento.

Estos elementos subjetivos de una situación clínica son para el paciente tan importantes o más que los parámetros biológicos y en realidad representan el objetivo fundamental de muchas de nuestras decisiones terapéuticas. Los PRO persiguen "medir" estos elementos subjetivos con la finalidad de saber objetivamente si un tratamiento es eficaz y hasta que punto. Para ello, la única posibilidad es preguntar directamente al paciente sobre ello; pero tanto la forma en que el clínico realiza la pregunta como la interpretación de la respuesta introducen elementos subjetivos ajenos al paciente. La utilización de cuestionarios que son completados por el paciente permite eliminar gran parte de estos elementos subjetivos y normalizar tanto las preguntas como las respuestas: estos son los instrumentos de medida. Son muchos los cuestionarios disponibles para evaluar diferentes circunstancias, algunos genéricos y otros centrados específicamente en un problema. Como cualquier otro instrumento de medida, estos cuestionarios deben someterse a unos controles de calidad durante su "fabricación", en este caso construcción. En el caso de los cuestionarios estos controles de calidad vienen dado por la evaluación de su fiabilidad ("reliability") y validez ("validity") (5).

Tras conocer que una escala es fiable y valida, el interés de un clínico se centra en saber si esta escala es capaz de detectar cambios clínicos. Este es lo que se conoce como sensibilidad al cambio ("responsiveness") del cuestionario. Más aún, al clínico no le importa simplemente saber si el cambio existe, sino sobre todo si dicho cambio es clínicamente relevante. Este último aspecto se objetiva determinando lo que se conoce como diferencia mínimamente importante ("minimal importante difference"; MID en la literatura anglosajona) $(6,7)$.

En este número de Anales de Medicina Interna, Rejas y cols. (8) nos dan la oportunidad de profundizar en este aspecto que aunque puede parecer puramente metodológico, tiene trascendencia para el clínico. Determinar la diferencia mínimamente importante no es una cuestión sencilla y su abordaje metodológico incluye dos visiones del problema: la primera, que es el abordaje mas clásico consiste en comparar los cambios obtenidos con una escala adicional completada por el paciente. El abordaje alternativo es puramente estadístico e incluye la evaluación de ciertas características de la distribución de datos, como la mitad de la desviación estándar o el error estándar de la media. Aunque las primeras son más fácilmente comprensibles, requieren utilizar otro cuestionario como referencia, lo que añade un elemento subjetivo adicional a la evaluación.

Rejas y cols. comparan en su trabajo estas metodologías para dos escalas utilizadas en urología, el LISAT-8 (cuestio- 
nario de satisfacción con la vida), especialmente utilizada par ala valoración del impacto de disfunción eréctil, y el BPHPIM (medida del impacto de la hiperplasia benigna de próstata), un instrumento de medida de CV específico para la hiperplasia prostática. Sus resultados claramente demuestran que las dos aproximaciones comparadas para medir la diferencia clínicamente significativa son prácticamente equivalentes $\mathrm{y}$, por tanto, pueden utilizarse igualmente para valorarla.

La incorporación de medidas del MID permite mejorar notablemente la interpretación de los PRO en estudios clínicos (6). Para afirmar que un tratamiento mejora la calidad de vida podemos basarnos en un cambio en una puntuación numérica, pero para el clínico no habituado a estas escalas dicho cambio numérico no tiene un significado claro. Incor- porando las MID, podemos saber, por ejemplo, el porcentaje de pacientes que experimenta un cambio superior al MID, lo que nos permite saber cuantos han experimentado una mejoría clínica real. Como podemos ver, los PRO no persiguen sustituir la clásica pregunta ¿ha mejorado usted? sino tratar de prever y responder a las preguntas del paciente ¿voy a mejorar? o ¿cuánto voy a mejorar?

\section{E. REY}

Servicio de Aparato Digestivo. Hospital Clínico San Carlos. Madrid

\section{Bibliografía}

1. Emery MP, Perrier LL, Acquadro C. Patient-reported outcome and quality of life instruments database (PROQOLID): frequently asked questions. Health Qual Life Outcomes 2005; 3: 12

2. Soto AJ. [Assessment of patient-reported outcomes: necessity or luxury?]. An Med Interna (Madrid) 2006; 23: 136-138.

3. Guidance for industry: patient-reported outcome measures: use in medical product development to support labeling claims: draft guidance. Health Qual Life Outcomes 2006; 4: 79.

4. Cella D, Yount S, Rothrock N, Gershon R, Cook K, Reeve B, Ader D, Fries JF, Bruce B, Rose M. The Patient-Reported Outcomes Measurement Information System (PROMIS): progress of an NIH Roadmap cooperative group during its first two years. Med Care 2007; 45: S3S11.

5. Coyne KS, Tubaro A, Brubaker L, Bavendam T. Development and validation of patient-reported outcomes measures for overactive bladder: a review of concepts. Urology 2006; 68: 9-16.

6. Brozek JL, Guyatt GH, Schunemann HJ. How a well-grounded minimal important difference can enhance transparency of labelling claims and improve interpretation of a patient reported outcome measure. Health Qual Life Outcomes 2006; 4: 69.

7. Revicki DA, Cella D, Hays RD, Sloan JA, Lenderking WR, Aaronson NK. Responsiveness and minimal important differences for patient reported outcomes. Health Qual Life Outcomes 2006; 4: 70.

8. Rejas J, Ruiz M, Pardo A. El error estandar de medida: ¿una alternativa a la diferencia minimamente importante para evaluar cambios en la salud autopercibida? An Med Interna (Madrid) 2007; 24: 415-420. 